\title{
Amalgamation based optical and colorimetric sensing of mercury (II) ions with silver@graphene oxide nanocomposite materials
}

\begin{abstract}
The article describes a facile method for the preparation of a conjugate composed of silver nanoparticles and graphene oxide (Ag@GO) via chemical reduction of silver precursors in the presence of graphene oxide (GO) while sonicating the solution. The Ag@GO was characterized by X-ray photoelectron spectroscopy, X-ray powder diffraction, and energydispersive $\mathrm{X}$-ray spectroscopy. The nanocomposite undergoes a color change from yellow to colorless in presence of $\mathrm{Hg}(\mathrm{II})$, and this effect is based on the disappearance of the localized surface plasmon resonance absorption of the AgNPs due to the formation of silver-mercury amalgam. The presence of GO, on the other hand, prevents the agglomeration of the AgNPs and enhances the stability of the nanocomposite material in solution. Hence, the probe represents a viable optical probe for the determination of mercury(II) ions in that it can be used to visually detect $\mathrm{Hg}$ (II) concentrations as low as $100 \mu \mathrm{M}$. The instrumental LOD is $338 \mathrm{nM}$.
\end{abstract}

Keyword: Silver nanoparticles; X-ray photoelectron spectroscopy; X-ray powder diffraction; Energy-dispersive X-ray spectroscopy; Localized surface plasmon resonance 